\title{
Integrated care through training - joint general practitioner/geriatric trainee clinics
}

\author{
Authors: Towhid Imam, Owen Ingram, Tom Wilson and Daniel Mullarkey
}

\section{Aims}

To address both a clinical need for integrated services and a training need, joint general practitioner (GP) and geriatric trainee clinics were created to allowing sharing of expertise and learning while improving the delivery of care to older patients.

\section{Methods}

A pilot joint clinic model in a GP practice in south-west London was developed. Two geriatric specialist trainees and two GP registrars ran six specialist geriatric clinics between August 2016 and February 2017. 25 patients with complex geriatric needs were identified by GP colleagues and referred to the clinics. Appointments were extended to 30 minutes to enable comprehensive geriatric assessment. Patient management plans were fed back to referring clinicians in the practice to enable whole practice learning. The project was evaluated by qualitative structured interviews with geriatric trainees, GP registrars and consultant and GP supervisors, and patient satisfaction questionnaires.

\section{Results}

The average age of patients was 78 years and common themes included cognitive impairment, urinary incontinence, polypharmacy and movement disorders. The feedback demonstrated learning for both GP registrars and geriatric trainees in clinical knowledge, service structure and collaborative approaches to patient care. The clinics were enjoyable and added significant value to training for both GP registrars and geriatric trainees. The GP registrars reported developing clinical knowledge and skills relating to the assessment of older patients. The geriatric trainees reported the development of communications skills and patient-focused approaches relating to primary care. The majority of patients were managed within primary care, with four referrals generated to secondary care. Six patient questionnaires were collected, with an average satisfaction score of 9.3 out of 10 .

\section{Conclusions}

There was qualitative evidence of learning from the GP and geriatric trainees, with good formal feedback from patients. This pilot project provided an exciting template to improve training of both GPs and geriatricians, improve the care of the older population and facilitate closer working between primary and secondary care. Barriers to implementation may include the existing pressures on services to take registrars out of secondary care and to use 30-minute appointments. Evaluation of costeffectiveness and patient outcomes should be investigated in future studies.

\section{Conflict of interest statement}

None declared. 